\title{
Ruptura de musculatura abdominal: relato de caso
}

\author{
Spontaneous rupture of abdominal musculature: case report
}

Retroceso de musculatura abdominal: informe de caso

Isadora Machado Pontes ${ }^{1}$, Sebastião Osmar Lourenço Neto ${ }^{*}$, Thiago Alves Hungaro ${ }^{1}$, Ana Carolina Zanin Sacoman Kurihara', Yoshio Sagata².

\section{RESUMO}

Objetivo: Relatar o caso de uma paciente com ruptura do músculo oblíquo e transverso, com consequente hematoma desencadeado por crise de tosse, cujo tratamento conservador proporcionou uma evolução favorável. Detalhamento do caso: Paciente sexo feminino, 63 anos, admitida no pronto socorro com equimose em quadrantes inferiores do abdome, com dois dias de evolução, após crise de tosse, culminando em quadro de dor de forte intensidade em flanco esquerdo. Realizada ultrassonografia de abdome inferior (US), sendo detectado líquido livre na cavidade abdominal e tomografia computadorizada (TC) de abdome superior e inferior que evidenciou ruptura dos músculos oblíquos e transverso do abdome com volumoso hematoma no local. Iniciou-se tratamento conservador com medicação sintomática e repouso absoluto. Houve boa evolução e após 45 dias, retornou assintomática e com melhora evidente das equimoses. Considerações Finais: $O$ caso relatado e a literatura pesquisada fornecem embasamento para a discussão da terapêutica de uma situação rara e pouco discutida que é o hematoma espontâneo da musculatura abdominal, e evidenciam que o tratamento conservador é capaz de obter resultados satisfatórios na resolução do quadro.

Palavras-chave: Ruptura espontânea, Músculos abdominais, Tratamento conservador, Hematoma.

\begin{abstract}
Objective: To report the case of a patient with rupture of the oblique and transverse muscle, with consequent hematoma triggered by a coughing crisis, whose conservative treatment provided a favorable evolution. Details of the case: Female patient, 63 years old, admitted to the emergency room with ecchymosis in lower quadrants of the abdomen, with two days of evolution, after coughing crisis, culminating in severe pain on the left flank. Ultrasonography of the lower abdomen (US) was performed, free fluid was detected in the abdominal cavity and computed tomography (CT) of the upper and lower abdomen that showed rupture of the oblique and transverse muscles of the abdomen with a large hematoma in the area. Conservative treatment was started with symptomatic medication and absolute rest. There was a good evolution and after 45 days, she returned asymptomatic and with evident improvement of bruises. Final Considerations: The reported case and the researched literature provide a basis for discussing the treatment of a rare and little discussed situation, which is the spontaneous hematoma of the abdominal muscles and show that conservative treatment is capable of obtaining satisfactory results in the resolution of the condition.
\end{abstract}

Keywords: Spontaneous rupture, Abdominal muscles, Conservative treatment, Hematoma.

${ }^{1}$ Centro Universitário Municipal de Franca Uni - FACEF, Franca - SP.

2 Hospital São Marcos, Morro Agudo - SP

*E-mail: neto_lourenco@hotmail.com 


\section{RESUMEN}

Objetivo: informar el caso de un paciente con ruptura del músculo oblicuo y transversal, con un hematoma de implicación, desencadenada por una crisis de tos, cuyo tratamiento conservador proporcionó una evolución favorable. Detalles del caso: Paciente femenina, de 63 años, ingresada en la sala de emergencias con equimosis en los cuadrantes inferiores del abdomen, con dos días de evolución, después de una crisis de tos, que culminó en un dolor intenso en el flanco izquierdo. Se realizó una ecografía de la parte inferior del abdomen (EE. UU.), que detectó líquido libre en la cavidad abdominal y una tomografía computarizada (TC) de la parte superior e inferior del abdomen que mostró ruptura de los músculos oblicuos y transversales del abdomen con un gran hematoma en el área. El tratamiento conservador se inició con medicación sintomática y reposo absoluto. Hubo una buena evolución y después de 45 días, regresó asintomático y con una mejora evidente de los moretones. Consideraciones Finales: El caso reportado y la literatura investigada proporcionan una base para discutir el tratamiento de una situación poco frecuente y poco discutida, que es el hematoma espontáneo de los músculos abdominales y indican que el tratamiento conservador es capaz de obtener resultados satisfactorios en la resolución de la condición.

Palabras clave: Ruptura espontánea, Músculos abdominales, Tratamiento conservador, Hematoma.

\section{INTRODUÇÃO}

A raridade do hematoma espontâneo da musculatura abdominal abre possibilidades de investigações etiológicas e diagnósticas, que contribuirão significativamente para compreendê-lo e tratá-lo de maneira precoce e efetiva.

Foi realizado levantamento de dados nas plataformas de pesquisa, e em virtude da escassez literária sobre ruptura espontânea dos músculos oblíquos e transverso do abdome, os artigos revisados para o presente estudo tratam do hematoma espontâneo da Bainha do Reto Abdominal (HSBRA), que é uma patologia benigna e rara, com incidência entre 46 e 69 anos, relacionada ao acúmulo de sangue na bainha deste músculo, decorrente da ruptura do mesmo ou de vasos epigástricos sem que tenha ocorrido trauma (HATJIPETROU A, et al., 2015).

Dessa forma, HSBRA é uma causa subestimada de dor abdominal aguda e que, apesar de a maioria ser autolimitada, pode evoluir rapidamente, incrementando o risco de morte, resultando em hemorragia e/ou choque circulatório, decorrentes do sangramento maciço (RAMOS GMD, et al., 2018).

É possível constatar que o diagnóstico precoce e o manejo clínico adequado são fatores determinantes para boa evolução do quadro e prognóstico do paciente.

Os principais fatores de risco relacionados a patologia são: sexo feminino, idade avançada, hipertensão arterial, doenças hematológicas, uso de anticoagulantes, doenças musculares degenerativas, gravidez, obesidade, cirurgias abdominais e quaisquer outras situações que ocasionam aumento da pressão abdominal, como tosse ou espirro (HATJIPETROU A, et al., 2015).

O diagnóstico é baseado em história clínica, realizada através de anamnese com aprofundada investigação de fatores de risco, aliados a exame físico e exames de imagem, como de ultrassonografia e tomografia computadorizada, sendo que a última apresenta maior especificidade e detalhamento em relação a localização, classificação e extensão do hematoma (BERNÁ JD, et al., 1996).

A maioria dos casos é autolimitada, justificando, portanto, a adoção de conduta conservadora, baseada em repouso, analgesia, ressuscitação fluídica intravenosa e transfusão sanguínea episódica (BERNÁ JD, et al., 2000).

Este estudo, tem como objetivo geral relatar o caso de uma paciente diagnosticada com hematoma abdominal por ruptura do músculo oblíquo e transverso, desencadeada por crise de tosse, cujo tratamento conservador, com repouso e analgesia, proporcionou uma evolução favorável do quadro. Apresenta como 
objetivos específicos, analisar as características clínicas, manejo terapêutico, conduta e prognóstico do paciente e relacioná-las à revisão de casos na literatura atual existente sobre o tema.

\section{DETALHAMENTO DO CASO}

Paciente sexo feminino, 63 anos, admitida no pronto socorro de um Hospital Geral, em um município do interior do Estado de São Paulo, com queixa de equimose em quadrantes inferiores do abdome, de início há dois dias.

Durante a anamnese, a paciente referiu estar em tratamento clínico para quadro de pneumonia adquirida na comunidade (PAC), em uso de levofloxacino e analgésicos simples, e que, no dia anterior ao surgimento dos hematomas, apresentou crises de tosse isoladas, culminando em quadro de dor súbita, de forte intensidade em região inferior do abdome, de maior intensidade em flanco esquerdo.

Paciente com antecedente de tabagismo, com carga tabágica de 40 anos/maço, hipertensão, sedentarismo e obesidade, sem histórico de discrasias sanguíneas, e com antecedentes de três cirurgias abdominais prévias, sendo estas duas cesarianas, há mais de 20 anos, e realizou ulcerorrafia há 22 anos. Sem outros antecedentes.

Ao exame, encontrava-se em bom estado geral, corada, hidratada, acianótica, afebril e eupnéica, saturando $92 \%$ em ar ambiente. Aparelho respiratório e cardiovascular sem evidências de alterações, encontrando-se dentro da normalidade. Ao exame abdominal observou-se à inspeção, presença de hematoma importante em quadrantes inferiores do abdome, de maior intensidade em flanco esquerdo. À palpação foi possível constatar massa mal delimitada em região de flanco esquerdo estendendo para fossa ilíaca esquerda de consistência endurecida e pouco dolorosa (Figura 1).

Figura 1 - Imagem mostrando a extensão do hematoma antes do tratamento.

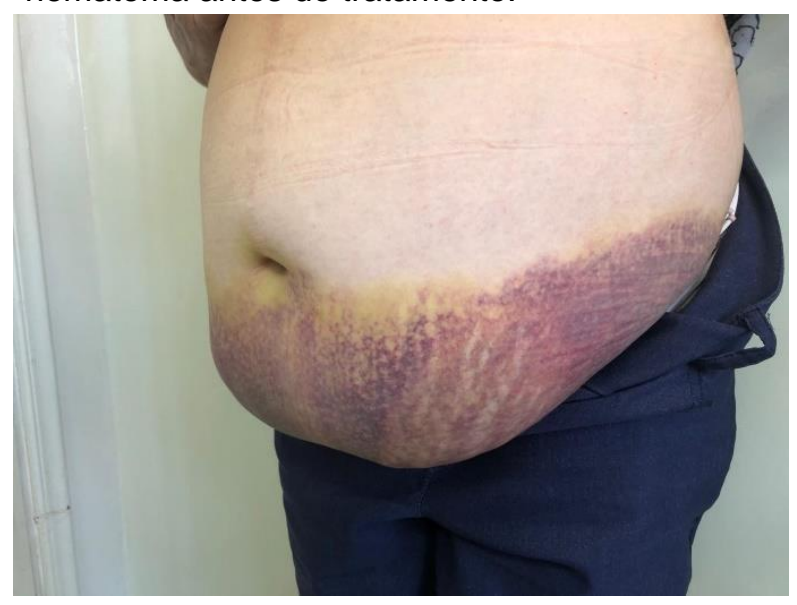

Fonte: Pontes IM, et al., 2020.

Baseado na sintomatologia e quadro clínico apresentado, iniciou-se investigação diagnóstica, sendo solicitado exames complementares, dentre eles: hemograma, que demonstrava anisocitose e hipocromia (hemoglobina 10,6 g/dl; hematócrito 30,6\%; volume corpuscular médio (VCM) 89,74 fL; hemoglobina corpuscular média (HCM) 31,09 pg; concentração da hemoglobina corpuscular média (CHCM) 34,64 g/dl e plaquetas $342.000 / \mathrm{mm}^{3}$ ), coagulograma mantendo parâmetros de normalidade (tempo de protrombina 13,9 segundos; razão normalizada internacional (RNI) 1,17 e tempo de tromboplastina parcial ativado 35 segundos), eletrólitos e função renal sem alterações.

Na sequência da investigação diagnóstica, foi realizado ultrassonografia de abdome inferior (US), sendo detectado líquido livre na cavidade abdominal. Em virtude da persistência de dúvida diagnóstica, a paciente 
foi inserida na Central de Regulação de Ofertas de Serviços de Saúde (CROSS) estadual e encaminhada para o hospital de referência, onde realizou tomografia computadorizada (TC) de abdome superior e inferior, que constatou ruptura dos músculos oblíquos e transverso do abdome com volumoso hematoma no local sem dissecção do plano fácil.

Desta forma, o HSBRA apresentado pela paciente foi caracterizado como do tipo I com base nos achados tomográficos e na condição de estabilidade hemodinâmica (Figura 2).

Figura 2 -Tomografia computadorizada evidenciando hematoma ruptura dos músculos oblíquos e transverso do abdome à esquerda com volumoso hematoma no interior dos mesmos.

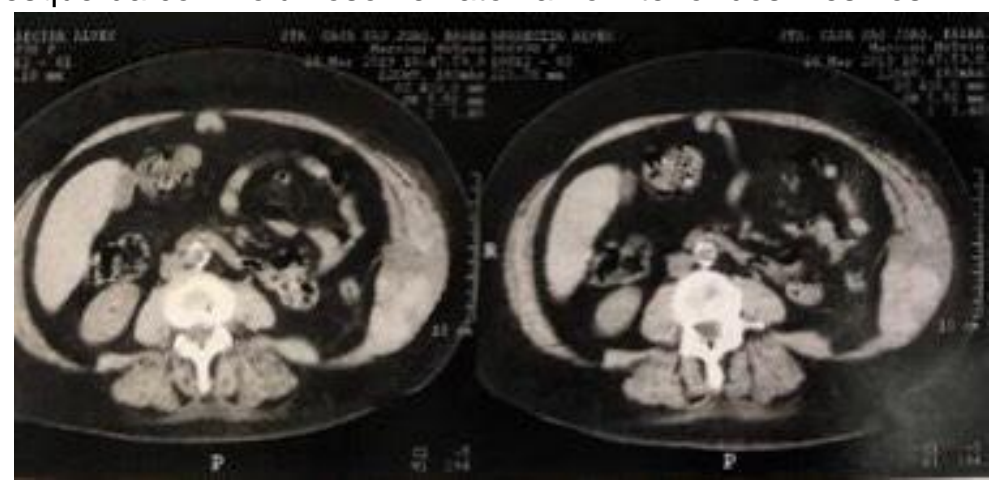

Fonte: Pontes IM, et al., 2020.

Em decorrência do diagnóstico precoce, foi possível realizar tratamento clínico conservador, inicialmente em ambiente hospitalar e posteriormente com seguimento ambulatório-domiciliar, composto por medicação sintomática e repouso absoluto, portanto evitando abordagem cirúrgica desnecessária naquele momento. Houve boa evolução do quadro clínico e após 45 dias do início do tratamento, paciente retornou assintomática e com melhora evidente das equimoses e assintomática (Figura 3).

Figura 3 - Imagem após o término do tratamento conservador mostrando evidente resolução do hematoma.

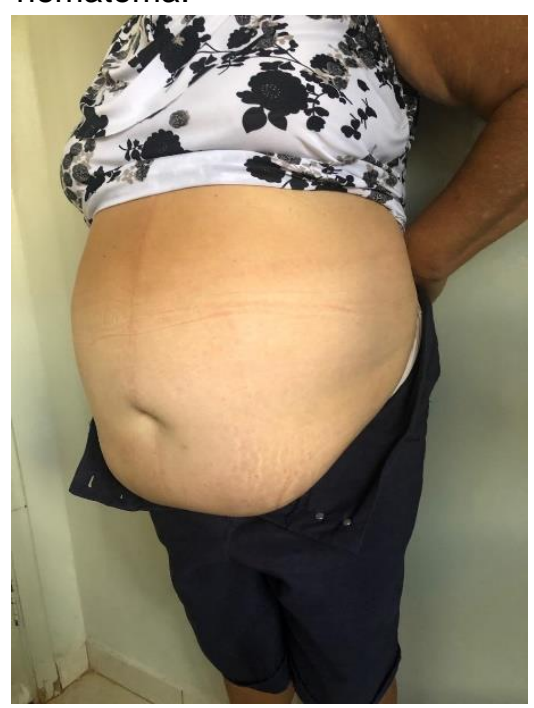

Fonte: Pontes IM, et al., 2020. 


\section{DISCUSSÃO}

Apesar de incomum, o hematoma da bainha do reto abdominal é conhecido desde os tempos de Hipócrates, Galeno e Leonardo da Vinci, há cerca de 2500 anos. O mesmo é caracterizado por um acúmulo de sangue na bainha deste músculo, em decorrência de lesão direta às fibras musculares ou danos às artérias epigástricas inferiores e/ou superiores, podendo ser de natureza aguda ou crônica (DUBE S, et al., 2018). Os casos descritos na literatura são, na maioria da vezes, decorrentes de eventos traumáticos sendo o relato dos não-traumáticos ou espontâneos escassos (VALENTIM LF, et al., 2005).

A artéria epigástrica inferior é mais importante para a circulação sanguínea da parede abdominal do que a artéria epigástrica superior, devido seu diâmetro externo. A artéria epigástrica inferior apresenta diâmetro de 3,4 $\mathrm{mm}$ e a artéria epigástrica superior é de 1,6 $\mathrm{mm}$. De acordo com a lei de Laplace, quando menor o raio, menor a tensão da parede. Por esse motivo, acredita-se que os casos de HSBRA ocorrem com mais frequência sob o umbigo, onde está localizada as artérias epigástricas inferiores (KARAPOLAT B, et al., 2019).

O sangramento proveniente dos vasos epigástricos superiores é unilateral e pode ser autolimitado, já o advindo dos vasos epigástricos inferiores, pode expandir progressivamente devido à ausência da bainha do reto abdominal, ganhando caráter bilateral que por vezes ultrapassa a linha média em direção à posterior, como o ocorrido no caso apresentado (ALLA VM, et al., 2010).

O HSBRA é cerca de 2 a 3 vezes mais comum no sexo feminino, devido à menor massa muscular da mulher e, na maioria, felizmente apresenta-se quadro benigno e autolimitado, com uma taxa de mortalidade de $4 \%$, podendo em alguns raros casos ser fatal (DUBE S, et al., 2018). Além disso, exibe prevalência maior na faixa etária entre os 50 e 60 anos, e sua incidência está aumentando devido ao crescente uso de anticoagulantes na população geral, pois, $69 \%$ dos casos de HSBRA estão relacionados com o uso desta classe de medicamentos, resultando em quadros com prognóstico variável de acordo com a etiologia (FITZGERALD JE, et al., 2009).

O hematoma do músculo reto abdominal pode ocorrer de maneira espontânea, o que é mais raro, ser resultado de trauma abdominal direto ou secundário a complicações pós operatórias, discrasias sanguíneas ou fatores gerais que levam ao aumento da pressão intra-abdominal (ONDER A, et al., 2011). Desta forma, inúmeros fatores de risco estão associados com o HSBRA, como aumento da idade, anticoagulação, aterosclerose, hipertensão, gravidez, tosse, doenças renais, contrações musculares e cirurgias da parede abdominal. No entanto, o fator predisponente de maior importância é a terapia de anticoagulação (FITZGERALD JE, et al., 2009).

O quadro clínico do HSBRA é composto principalmente por dor abdominal súbita (84-97\%) e por massa palpável em parede abdominal (63-92\%), além disso, o paciente pode apresentar sintomas inespecíficos como, náuseas, taquicardia, hipotensão e febre. A dor é de início agudo, de forte intensidade, sem fatores de melhora e com piora à movimentação (DUBE $\mathrm{S}$, et al., 2018). As equimoses abdominais, semelhantes às evidenciadas no quadro desta paciente, estão presentes em apenas cerca de $17 \%$ dos casos e indicam a extensão extraperitoneal do hematoma (WATANABE A, et al., 2014). Acompanhando o quadro álgico pode haver presença de uma massa abdominal palpável que não ultrapassa a linha média do abdome e, que diferente de uma massa intraperitoneal, ao elevar os membros ou flexionar a cabeça tensionando o músculo reto, ela permanece palpável e visível, caracterizando o sinal de Fothergill (OSINBOWALE O e BARTHOLOMEW JR, 2008). Além deste, podem estar presentes o sinal de Carnet referente ao aumento da sensibilidade e dor na região do hematoma ao tensionar o músculo e ao final do curso da lesão, os sinais de Gray Turner e Cullen, descritos respectivamente como equimoses nos flancos e periumbilical e apresentados pelo paciente do caso (DUBE S, et al., 2018).

Como o HSBRA encontra-se em menos de $2 \%$ dos pacientes com abdome agudo, não é considerado um dos diagnósticos iniciais de dor abdominal aguda, e o diagnóstico clínico é visto como difícil, principalmente em salas de emergência, passando muitas vezes despercebido (HATJIPETROU A, et al., 2015). Por isso, é 
crucial saber reconhecer a clínica apresentada por esses pacientes e diferenciá-la de outras causas de dor abdominal aguda.

O diagnóstico da HSBRA é obtido pela combinação da suspeição clínica com exames de imagem. A ultrassonografia é o exame inicial de escolha, utilizado em casos de dor abdominal aguda devido seu baixo custo e sensibilidade em torno de $80-90 \%$, no entanto, pode fornecer informações limitadas devido peculiaridades do paciente como tecido adiposo avantajado, além de ser um exame examinador dependente (ONDER A,et al., 2011). A tomografia computadorizada (TC) é o padrão ouro para diagnóstico e pode alcançar sensibilidade de $100 \%$, além de fornecer informações adicionais como localização, extensão e origem do hematoma. Os achados típicos da TC são: massa muscular com padrão misto, com níveis fluído-líquido devido o sangramento e massa isodensa com áreas hipodensas. Em adição, deve ser feito Angiotomografia na qual pode ser visto sangramento arterial ativo evidenciado como área focal de maior densidade dentro do hematoma (PIERRO A, et al., 2018).

Quando o paciente se apresenta hemodinamicamente instável, uma cirurgia é planejada ou drenagem percutânea, a TC é importante. Com base na TC é possível classificar a HSBRA em três tipos (DUBE S, et al., 2018):

Tipo I: coleção unilateral e intramuscular que não disseca o plano facial e não causa instabilidade hemodinâmica.

Tipo II: coleção bilateral com pequena dissecção entre o músculo, fáscia transversal e peritônio e que causam instabilidade hemodinâmica mínima.

Tipo III: coleção mais severa, bilateral com dissecção entre a fáscia transversal e o músculo, causando instabilidade hemodinâmica. Pode exigir intervenção cirúrgica imediata.

Desta forma, este exame determina um aumento significativo na acurácia diagnóstica, facilitando realização de conduta resolutiva com maior precocidade e ajustada para cada caso e paciente. Ampliando a avaliação diagnóstica ou nos casos de indisponibilidade da TC, pode-se utilizar de métodos complementares de menor acurácia no diagnóstico da HSBRA como, a radiografia simples de abdome em decúbito dorsal e a ultra-sonografia abdominal. Tais exames podem demonstrar respectivamente, imagem hipotransparente contrastando com as alças intestinais e imagem ecogênica fusiforme parietal (VALENTIM LF, et al., 2005).

O manejo frente a um quadro de HSBRA pode ser dar com base na sua classificação tomográfica, desta maneira, hematomas tipo 1 geralmente não requerem hospitalização e possuem resolução em média de 30 dias, já nos tipos 2 e 3, a internação é obrigatória e mandatória a fim de evitar complicações (DUBE S, et al., 2018).

A conduta conservadora com repouso absoluto ou relativo, reposição de líquidos e analgesia é, na maioria dos casos, eficaz para resolução do quadro sendo o tratamento cirúrgico indicado apenas nos casos de falha ao tratamento conservador ou instabilidade hemodinâmica, o que representa a minoria dos casos. (ONDER A, et al., 2011).

Quando necessária a abordagem cirúrgica procede-se com evacuação do hematoma por drenagem seguida da hemostasia do vaso sangrante, no entanto, a mortalidade dos pacientes que necessitam deste tipo de intervenção é alta (ONDER A, et al., 2011).

Além disso, a abordagem cirúrgica mesmo quando devidamente indicada, apresenta riscos inerentes à qualquer procedimento, como desenvolvimento de complicações pós-operatórias, entre elas, a infecção. Sendo esta, uma grande contribuinte para taxa de mortalidade do procedimento variar entre 4 a $18 \%$ (VALENTIM LF, et al., 2005).

A embolização arterial do vaso hemorrágico é o tratamento atualmente preferido em casos de alto risco, que não respondem ao tratamento conservador, instáveis hemodinamicamente e que apresentam sangramento incontrolável, ou hematoma aumentando continuamente de tamanho, ou infecção complicada,

REAS/EJCH | Vol.Sup.n.49 | e3427 | DOI: https://doi.org/10.25248/reas.e3427.2020 Página 6 de 7 
e pacientes que apresentam alterações peritoneais graves irritação ou sinais de síndrome do compartimento abdominal (KARAPOLAT B, et al., 2019).

A cirurgia é usada quando ocorre falha na embolização ou a drenagem do hematoma é necessária, devido a complicações como infecção ou síndrome de compartimento abdominal. No entanto, essa técnica é demorada, cara, nem sempre está disponível nos serviços médicos e nem sempre o vaso hemorrágico é identificado. Este procedimento também está associado a complicações como nefropatia induzida por contraste ou sangramento no local da punção (RIMOLA J, et al., 2007). Assim, na maioria dos casos de hematoma abdominal a evolução é autolimitada e a abordagem conservadora é eficaz na resolução do quadro. Os casos de complicações estão diretamente relacionados com as comorbidades do paciente, as taxas de mortalidade variam de $4 \%$ a $25 \%$ (SELMA AB e GENESE T, 2019).

Apenas um pequeno número de pacientes portadores de HSBRA complicada necessita de abordagem cirúrgica com drenagem do hematoma e hemostasia do vaso sangrante. Considerando o apresentando nesta pesquisa, o diagnóstico precoce é fator determinante na boa evolução do quadro e na conduta adotada, pois, consegue impedir intervenções cirúrgicas desnecessárias e garante maior sucesso ao tratamento conservador, reduzindo o custeio do tratamento para as instituições assistentes.

\section{REFERÊNCIAS}

1. ALLA VM, et al. Spontaneous Rectus Sheath Hematoma. The Western Journal of Emergency Medicine, 2010; 11(1): 76-79.

2. BERNÁ JD, et al. Rectus sheath hematoma: diagnostic classification by CT. Abdominal Imaging, 1996; 21(1): 62-64.

3. BERNÁ JD, et al. Conservative treatment of large rectus sheath hematoma in patients undergoing anticoagulant therapy. Abdominal Imaging, 2000; 25(3): 230-234.

4. DUBE S, et al. Rectus sheath hematoma: a potentially life threatening complication following caesarean delivery. International Journal of Reproduction, Contraception, Obstetrics and Gynecology, 2018; 7(10): 4289-4291.

5. FITZGERALD JE, et al. The changing nature of rectus sheath haematoma: case series and literature re view. Internatinal Journal of Surgery, 2009; 7(2): 150-154.

6. HATJIPETROU A, et al. Rectus sheath hematoma: A review of the literature. International Journal of Surgery, 2015; 13: $267-271$.

7. KARAPOLAT B, et al. Conservative Treatment of Spontaneous Rectus Sheath Hematomas: Single Center Experience and Literature Review. Emergency Medicine International, 2019; 2019:1-7.

8. ONDER A, et al. A Conservative Approach to Rectus Sheath Haematomas. Eletronic Journal of General Medicine, 2011; 8(3): 224-228.

9. OSINBOWALE O, BARTHOLOMEW JR. Rectus sheath hematoma. Vascular Medicine, 2008; 13(4): 275-279.

10. PIERRO A, et al. Spontaneous rectus sheath hematoma: The utility of CT angiography. Radiology Case Report, 2018; 13: 328-332.

11. RAMOS GMD, et al. Rectus sheath hematoma manifesting as hemorrhagic shock. Medical Illustration, 2019; 14(1):181-182.

12. RIMOLA J, et al. Percutaneous Arterial Embolization in the Management of Rectus Sheath Hematoma. American Journal of Roentgenology, 2007; 188(6): 497-502.

13. SELMA AB, GENESE T. Spontaneous Rectus Sheath Hematoma: An Uncommon Cause of Acute Abdominal Pain. American Journal of Case Reports, 2019; 20: 163-166.

14. VALENTIM LF, et al. Hematoma Espontâneo do Múculo Reto Abdominal. Rev Col Bras Cir, 2005; 32(3): 165-166.

15. WATANABE A, et al. Spontaneous Rectus Sheath Hematoma Associated With Low Dose of Acetylsalicylic Acid. ABCD Arq Bras Cir Dig, 2014; 27(1): 84-85. 